\title{
Frailty indexes, screening instruments and their application in Belgian primary care
}

\author{
E. Sieliwonczyk, S. Perkisas, M. Vandewoude
}

Department Geriatrics, University of Antwerp, ZNA St Elisabeth Leopoldstraat 26, 2000 Antwerpen, Belgium

\begin{abstract}
Objectives: The complex and expensive medical care for a rising number of older patients presents a significant challenge to the health care system. Identifying cost-effective preventive interventions and systematically applying them in the elderly population could help address this challenge. Frailty assessments could prove to be valuable tools by identifying at-risk individuals to which these interventions would be offered. This review seeks to provide the reader with an overview of frailty and explain how frailty assessments could contribute to daily practice.

Methods: PubMed was searched for articles concerning frailty assessment (July 2013). Articles discussing prominent frailty models and articles primarily focused on comparing frailty assessments in the homedwelling population were used for this article. Domus Medica was searched for guidelines concerning the use of frailty in Belgian primary care.

Results: Several notable models of frailty are summarized and discussed to provide the reader with an overview of available frailty assessments. Frailty screening modalities in primary care are discussed, as well as the current recommendations for the use of frailty assessments in Belgian primary care. The advantages of a systematic frailty assessment in primary care and other settings are highlighted.

Conclusion: This article recommends the assessment of frailty status as a screening tool for the evaluation of the older person in primary care. An overview of available frailty models is offered for this purpose. A consensus should be reached on which model is most appropriate. The screening for frailty promotes early intervention and timely involvement of specialists with the purpose of avoiding unfavourable outcomes, such as death or disability.
\end{abstract}

Keywords: Geriatric medicine, Frailty, Frailty models, Primary care, Screening instruments

\section{Introduction}

The condition of frailty is defined as a state wherein the individual is more likely to experience adverse events, such as increased dependency and/or death, when exposed to a stressor. The cause of frailty is sought in a loss of physiological reserve. The body's physiological reserves decline as a consequence of aging-related changes. Reparative mechanisms in the body attempt to undo these changes. When these mechanisms fail, the individual acquires deficits and insufficiently repaired insults expressed as symptoms, signs, laboratory abnormalities, diseases, and disabilities. In geriatric medicine frailty is used to explain the differences in physiological resilience between members of same age groups. While many diseases become more common with age, many older adults are fit and quite capable of compensating for these stressors. The frail patients however are at risk

Correspondence to: Ewa Sieliwonczyk, Department Geriatrics, University of Antwerp, ZNA St Elisabeth Leopoldstraat 26, 2000 Antwerpen, Belgium. Email: ewa.sieliwonczyk@gmail.com of significant decompensation upon exposure to the same stressors. ${ }^{1,2}$

Although this concept of frailty is widely agreed upon, the best model for measuring frailty remains to be established. Many models have been proposed for application in the scientific and (less frequently) the clinical setting. Two approaches for measuring the physical domains of frailty are most frequently listed. ${ }^{1}$ The frailty phenotype model defines the frail, pre-frail and robust state based on the evaluation of five criteria: exhaustion, low activity, weight loss, slowness and weakness. ${ }^{3}$ The second model, the deficit model, measures the individual's deficits in normal functions. The deficits are then summarized in a frailty index. The frailty models derived from the deficit model often employ measurements derived from the comprehensive geriatric assessment (CGA). ${ }^{2}$

Recently, frailty is being seen as a multidimensional concept and newer models attempt to integrate the physical, cognitive, psychological and social dimensions of frailty. ${ }^{4,5}$ The relationship between these frailty dimensions is complicated. Several determinants exist across more than one dimension. 
For example, there is an overlap in the psychomotor symptoms of psychiatric diseases, most notably depression, and physical frailty. Both lower cognition and greater depressive symptoms have been shown to be associated with physical frailty. ${ }^{6,7}$ Social vulnerability has also been proven to be higher among the frail. ${ }^{8}$ Both lower cognition and social vulnerability can independently predict adverse outcomes associated with physical frailty. ${ }^{7,8}$ This suggests that the concept of frailty should be viewed as holistic and integrate these four dimensions. This view is congruent with clinical practice, where a holistic view of the patient is encouraged.

Successful identification of frail individuals could contribute greatly to care for the older person. If the at-risk group can be identified before decompensation occurs, preventive measures can be taken to avoid adverse outcomes. Several effective interventions to reduce the degree of frailty have been validated, such as exercise, vitamin D supplementation, protein-calorie supplementation and reduction of polypharmacy. ${ }^{1}$

Frailty assessments can also find their use in inpatient settings, as an assessment of the current status and general health of the patient. Through the use of frailty physicians are better equipped to make decisions regarding treatment options and have a better outlook on the patient's prognosis.

Frailty is also a major contributor to healthcare expenses, which can only be expected to rise in the future, given the aging of the population. In Belgium alone, the proportion of the population over 80 years old has increased from $4.12 \%$ in 2004 to $5.65 \%$ in 2013 and there is no reason to assume this trend will stop in the coming years. ${ }^{9}$ By identifying the frail individuals and applying targeted preventive interventions it should be possible to reduce the adverse events as well as their associated costs.

This review will discuss some of the different models of frailty and proposed measurement methods, with an emphasis on the frailty phenotype model, the frailty index model and the Tilburg Frailty Indicator. The models discussed in this article stand as examples of certain perspectives on frailty, from which many derivative models have been constructed. Both the theoretical aspects of these models and the clinical feasibility of the proposed measurements in daily practice are discussed in the article. The goal of this review is to identify and discuss the most prominent frailty models in consideration of their application within the Belgian healthcare system.

\section{Methodology}

PubMed was used to find relevant reviews using the terms 'frailty' AND ('index' OR 'models' OR 'measurement'). Of 131 results, 49 reviews were identified as potentially relevant to this article, based on a screening of the titles and abstracts. The search was primarily focused on reviews discussing and comparing the different frailty models and studies on the application of frailty screening tools in the homedwelling population. Articles were considered if their primary subject matter concerned frailty, if they were available in either Dutch or English and if a full version of the article was available.

This article is mainly based on the most recent consensus on frailty. ${ }^{1}$ Several articles have been selected from the reference list in the consensus article. Additional articles were selected from other references and from consultations with experts on the field of frailty.

Domus Medica was used to find recommendations for the use of frailty in Belgian primary care. One guideline concerning fall-prevention recommended the use of frailty, this guideline has been included. ${ }^{10}$

\section{Results \\ Frailty prevalence and epidemiology}

The prevalence of frailty can vary greatly between studies, most likely because of the many different definitions of frailty in scientific literature. The reported frailty prevalence rate varies between $4 \%$ and $59.1 \%$, as established by a recent study of crosssectional data gathered in 21 community-based cohorts. ${ }^{11}$ The weighted average prevalence rate has been established as $9.9 \% .{ }^{11}$ Frailty is known to increase with advancing age. ${ }^{1-3}$ Different studies show that women score higher on frailty assessments, ${ }^{1,12}$ even though the mortality rate in women is lower than in men. Variations in prevalence have been observed in populations of different race and ethnicity, with a higher prevalence of frailty in southern European, Hispanic and African-American populations. ${ }^{12,13}$

\section{General characteristics of frailty models}

Many models of frailty have been proposed over the years. These models try to define the term frailty and construct a number of measurement tools to establish the frailty status of an individual. The adverse outcomes the models attempt to predict are usually defined as death, hospitalizations and disability, measured by deficits in activities of daily living (ADL) or instrumental activities of daily living (IADL). The frailty models are very variable, some only focusing on the physical aspects of frailty, others expanding the concept to psychological, cognitive and social dimensions.

To maintain the validity of proposed models, several criteria have been suggested for a successful definition of frailty ${ }^{14}$ (Table 1). The frailty model should include multifactorial determinants, be 
Table 1 The necessary criteria for any successful definition of frailty, as suggested by Rookwood et al. ${ }^{13}$

\begin{tabular}{ll}
\hline Criterion & Meaning in frailty \\
\hline Content validity & The definition is dynamic, includes multiple determinants and is useful in different situations. \\
Construct validity & Frailty is more common in women and advancing age and it is related to disability. \\
Criterion validity & Frailty predicts adverse outcomes, including mortality. \\
\hline
\end{tabular}

dynamic (include change over time) and useful across different contexts. It should also supersede other successful definitions and be computationally traceable. These characteristics are summarized as the content validity criterion. The second criterion, construct validity, explores the adherence of the proposed model to the basic assumptions about frailty, such as the relation of frailty to age, gender and disability. The model should also make accurate predictions, to fulfill the final criterion, criterion validity.

While many models of frailty have been validated in this manner, the tools used in these studies vary greatly. Some models rely on questionnaires, administered by a physician or self-reported. Many models require specific tools. Some measurements do not require specific tools, but can nonetheless be difficult to perform in the primary care setting. These practical requirements are important determinants of the feasibility of the measurement in clinical practice, especially in primary care.

\section{The frailty phenotype model}

The frailty phenotype model is a categorical model, which distinguishes three frailty states, the frail, prefrail and robust state. In this model frailty is operationalized on a syndromal level, meaning that frailty is defined by the presence or absence of specific deficits (see Table 2). This model has been validated to predict greater mortality in the pre-frail and, more strongly, in the frail group in the Cardiovascular Health Study in the United States. ${ }^{3}$ The rise in mortality was still significant upon correction for comorbidities and ADL deficits. This definition of frailty is much more specific than the broad definition proposed by the deficit model, as it is based on only five possible deficits, all of which are related to physical frailty.

The assessment of frailty is based on a scoring of five possible deficits: slow walking speed, weakness, declining activity levels, shrinking and exhaustion. The measurement of gait speed requires a $4 \mathrm{~m}$ minimum distance, which is not always available given the limited working space of some primary physicians' offices. ${ }^{15} \mathrm{~A}$ dynamometer is used to assess for weakness. This device measures the hand grip pressure. The subject is asked to grip the dynamometer as tightly as possible, the inability to generate a sufficiently high handgrip pressure is a possible sign of frailty. Few primary care physicians have disposal of tools such as dynamometers. The other deficits are assessed by questionnaires and patient report.

The frailty assessment used in the Cardiovascular Health Study is not easy to implement in primary care. The required measurements are difficult to perform, time consuming and require specific tools. The cut-off numbers for the assessment of the deficits may depend on the population. Therefore the model

Table 2 The evaluation of frailty status according to the frailty phenotype model. Five clinical deficits are assessed to identify the individual frailty status as frail, pre-frail or robust ${ }^{3}$

Frailty phenotype assessment used in study of frailty in older adults, Fried $2001^{3}$

\begin{tabular}{|c|c|c|}
\hline Deficit & Measurement & Scoring \\
\hline 1. Slow walking speed & Time to walk 20 feet (approximately $5 \mathrm{~m}$ ) & $\begin{array}{l}\text { Present if scored in the lowest } 20 \% \\
\text { at baseline (adjusted for gender and } \\
\text { height) }\end{array}$ \\
\hline 2. Weakness & $\begin{array}{l}\text { Maximum pressure generated on } \\
\text { handgrip dynamometer }\end{array}$ & $\begin{array}{l}\text { Present if scored in the lowest } 20 \% \\
\text { at baseline (adjusted for gender and } \\
\text { Body Mass Index) }\end{array}$ \\
\hline 3. Declining activity levels & $\begin{array}{l}\text { Kilocalories expended per week based on the } \\
\text { assessments by Siscovick et al. } .^{32} \text { and Taylor et al. }{ }^{33}\end{array}$ & Present if scored in the lowest $20 \%$ \\
\hline 4. Shrinking & $\begin{array}{l}\text { Unintentional weight loss in the last year based } \\
\text { on self report }\end{array}$ & $\begin{array}{l}\text { Present if weight loss of } \geqslant 4.5 \mathrm{~kg} \\
\text { or } \geqslant 5 \% \text { body weight }\end{array}$ \\
\hline 5. Exhaustion & $\begin{array}{l}\text { Two statements from CES-D scale: 'everything } \\
\text { I did was an effort' and 'I could not get going' }\end{array}$ & $\begin{array}{l}\text { Present if patient reports } \\
\text { agreeing with either statement a } \\
\text { moderate amount of time or most } \\
\text { of the time last week }\end{array}$ \\
\hline $\begin{array}{l}\text { Assessment: } \\
\cdot \geqslant 3 \text { deficits: frail } \\
\cdot 1-2 \text { deficits: prefrail } \\
\text { - No deficits: robust }\end{array}$ & & \\
\hline
\end{tabular}

Note: *CES-D: Center for Epidemiological Studies Depression Scale, a short self-report scale for measuring depressive symptoms. 
cannot be implemented without prior literature search or a population study.

Other categorical models, derived from the frailty phenotype model, have been constructed to provide a simpler screening tool. A notable example is the FRAIL questionnaire, a very simple screening tool, comprised of five questions that can also be selfadministered (Table 3). This questionnaire has been proven to predict mortality and increased ADL and IADL disability over 9 years of follow-up, even when the individuals with ADL disability were excluded. ${ }^{13}$

Other studies recommend using a single component to screen for frailty, such as grip strength ${ }^{16}$ or walking speed, ${ }^{17}$ although it may be hard to meet the practical requirements of these measurements in the primary care setting.

\section{The deficit model}

The deficit model defines frailty as an age-associated accumulation of deficits. As opposed to the frailty phenotype model, in the deficit model the possible deficits are not strictly defined and can come in many forms. The deficit model can be used with almost any health related variable. The ratio of accumulated deficits and the total number of measured variables is an indicator of the risk status of the patient. Thus frailty is expressed on a continuum, rather than the categorical classification employed in the frailty phenotype model. ${ }^{2,3}$ The continuous frailty index has shown advantage in discriminatory ability for people with moderate to severe frailty, but overall both models show notable statistical convergence. ${ }^{12}$

The frailty assessments based upon the deficit model often employ tools from the CGA. The CGA explores the physical, psychiatric, cognitive and social determinants of health with a particular focus on the problems in the geriatric population, such as fall risk, comorbidities and polypharmacy. The use of the CGA is widespread and allows a multidisciplinary approach to the complex health issues of the older person. Guidelines for the use of the CGA are available in several languages, including English, Dutch and French.

Most of the data for the CGA can be acquired through patient interview, history and questionnaires. Supplementary tests can be included if needed, such as blood analyses or bladder function tests. The time demand for the completion of an assessment depends on the number and nature of the selected variables. More extensive assessments include more variables of the CGA and can be used to evaluate patients with a high risk of frailty in situations where they would benefit from a detailed assessment, e.g. in case of non-urgent hospital admission. Assessments including fewer variables can be used to screen for frailty in the general population.

\section{The multidimensional model}

Both the frailty phenotype model and the original deficit model place a significant emphasis on the physical losses in the elderly. The Tilburg Frailty Indicator (TFI) has been developed as an alternative to these models. It is intended to be linked with an integral conceptual frailty model, an approach that recognizes the multifactorial nature of frailty. It is meant to lead towards a health based, integrative approach, rather than the organ and disease based approaches taken by previous models. It defines frailty as a dynamic state with losses in three domains of human functioning: physical, physiological and social. The TFI is a self-administered questionnaire assessment of functioning within these domains. It is comprised of 15 items and requires in average 14 minutes to administer. ${ }^{5}$ The total score is measured on a scale from 0 to 15 with the cut off for a positive test result at 5. Both an English and a Dutch version are available.

The Groningen Frailty Indicator (GFI) is another example of a multidimensional frailty model. It has been constructed as a response to several drawbacks of other multidimensional frailty models. The main drawbacks are described as the limitation of the use

Table 3 The assessment of frailty status by the administration of the five-item FRAIL questionnaire ${ }^{12}$

\begin{tabular}{|c|c|c|}
\hline \multicolumn{3}{|c|}{ The FRAIL questionnaire ${ }^{12}$} \\
\hline Indicator & Question & Scoring \\
\hline Fatigue & $\begin{array}{l}\text { How much time did you feel tired during } \\
\text { the last } 4 \text { weeks? }\end{array}$ & All the time/most of the time: 1 point \\
\hline Resistance & $\begin{array}{l}\text { Do you have any difficulty walking up } 10 \text { steps } \\
\text { alone without resting and without aids? }\end{array}$ & Yes: 1 point \\
\hline Ambulation & $\begin{array}{l}\text { Do you have any difficulty walking several } \\
\text { hundred yards alone and without aids? }\end{array}$ & Yes: 1 point \\
\hline Illness & List of 11 illnesses & $\geqslant 5$ of listed illnesses: 1 point \\
\hline $\begin{array}{l}\text { Loss of weight } \\
\text { Assessment: } \\
\text { - 3-5 points: frail } \\
\text { - 1-2 points: pre-frail } \\
\text { - } 0 \text { points: robust }\end{array}$ & Self reported weight decline of $\geqslant 5 \%$ within 12 months. & Yes: 1 point \\
\hline
\end{tabular}


of the models to certain populations only (such as home-dwelling), the lack of both professional and self-assessed versions; they do not assess for disability and do not allow for grades of frailty. The main aims of the GFI are to address these drawbacks and to provide a short and feasible screening instrument.

The GFI is a 15 -item screening instrument, which exists in both a professional and a self-assessment version, with both English and Dutch versions available. As a multidimensional model, it measures deficits in several domains: the physical (mobility functions, multiple health problems, physical fatigue, vision and hearing), the cognitive (cognitive dysfunction), social (emotional isolation), and psychological (depressed mood and feelings of anxiety) domain. If the item is scored positive, the individual's score is increased by 1 point. The total score ranges from 0 to 15 , with a score of 4 or more representing moderate to severe frailty. ${ }^{18}$

\section{Discussion}

Frailty assessments can be employed as a screening tool to detect at risk individuals in community dwelling elderly. Many validated frailty models could be used for this purpose, ranging from self-administered questionnaires to examinations performed by a physician. The best tool for such a screening program remains to be determined. ${ }^{19}$ Because of the extremely multifactorial nature of frailty it is difficult to construct a model that is both highly accurate and easy to use. The perfect model may never be found, but many current models are sufficiently validated to implement them in clinical practice.

A recent study ${ }^{15}$ has reviewed the literature, searching for the most appropriate screening tool in primary health care. The TFI was recommended because of the simplicity of administration of this measurement and the validation of the model as a predictive score for adverse events after 1 year of follow-up. However, because the TFI is self-administered, it is a very subjective measurement that is vulnerable to bias. It can be influenced by many factors, such as the individual emotional state at the time of assessment, or the social desirability bias. This may be a relevant disadvantage to the use of this assessment.

Another study comparing the GFI to a frailty assessment derived from the deficit model recommends the combination of both assessments. This study shows that the GFI and the Frailty Index only moderately overlap in identifying frailty in community-dwelling elderly. ${ }^{20}$ This finding suggests that both screening tools may identify different frailty subgroups. This finding is not unexpected, given the multidimensional nature of frailty. ${ }^{21}$ If this is the case, the combination of both screening tools seems to be a valuable option. To provide optimal proactive primary care, the authors suggest an initial FI screening in routine healthcare data, followed by a GFI questionnaire for patients with a high FI score or otherwise at high risk as the preferred two-step frailty screening process in primary care. This twostep screening is proposed in other settings as well, such as the assessment of cancer patients. ${ }^{22}$

In the previous sections we have considered the holistic nature and multidimensionality of frailty. The holistic view brings up several new possibilities for the clinical use of frailty. For example, physical determinants could be used to help evaluate patients with cognitive or psychological impairments. This use of frailty has already been examined, although more research is encouraged, especially in the psychiatric setting. ${ }^{6}$ Frailty has been shown to successfully predict the outcome in patients with cognitive decline. ${ }^{7}$ Frailty could also be used to identify patients with psychiatric symptoms or cognitive impairment. The frailty status is associated with both depressive symptoms and cognitive changes. ${ }^{6,23}$ Some of the factors contributing to the development of both conditions seem similar for both frailty and cognitive decline. These findings suggest that a psychiatric and cognitive function evaluation may be warranted in patients with high frailty scores in assessments based on physical frailty models.

Frailty could be used to build a structured model of care for the elderly focused on preventive primary care medicine. ${ }^{19}$ The main focus of preventive medicine in Belgium is on the prevention of cardiovascular and oncological disease in the 45-75 year age group. Current preventive medicine offered to elderly patients is limited to fall prevention. The fall prevention guideline recommends the use of the frailty phenotype model namely the FRAIL questionnaire to identify frail patients in the age group $>65$ years old.

Further measures could be offered to these patients to improve their frailty status as well as a more detailed evaluation for geriatric, cognitive, psychiatric and social issues. An integration of the frailty assessment and the follow-up strategies for frail patients in the GMD (Globaal Medisch Dossier, global medical file) would likely improve the implementation of this intervention and provide a platform for communication between the patient, the primary care physician and the specialized care providers (geriatrician, hospital, neurologist, psychiatrist,...). The GMD is a medical file containing the medical data of a patient, such as the history of surgery, chronic diseases and running treatments. The goal of the GMD is the improvement of the individual patient follow up and communication between medical professionals. The patients can initiate the GMD at their own request during a consultation with 
the primary physician. The GMD can also include a prevention module.

A running project in Toulouse with the Gérontopole $^{24}$ examines the application of frailty measurement in primary care. This study is an interesting example of setting up a frailty screening program and the results of this study will without a doubt provide a lot of information about the practical aspects and efficiency of implementing frailty screening programs.

Frailty assessments have an important application beyond the scope of primary care. The care for the older person in the hospital setting is a complex matter. Issues typical to this population, such as multimorbidity and polypharmacy, complicate care. Frailty could help assess these vulnerable patients ${ }^{25}$ and help guide care and deliberate the patient's outcomes. Frail patients undergoing major elective surgery have been shown to have an increased risk of institutionalisation. ${ }^{26}$ When a CGA-frailty assessment is preformed after acute admission to the hospital, the survival chances of the patient improve. ${ }^{27}$ To select patients for CGA assessment in the emergency room setting, other, more easily administered assessments may be used. ${ }^{28}$ The use of frailty has been proposed as well for the assessment of critical care patients, ${ }^{29}$ cancer patients ${ }^{30,31}$ and home-care patients. ${ }^{31}$

\section{Conclusion}

The use of frailty would allow clinicians to introduce early interventions in the care for the older person, thus shifting the care towards a preventive course and thereby reducing the adverse outcomes as well as the public costs. Standardization of the concept of frailty and its measurement tools could build a platform for communication about the complex care for the older person between clinicians of different specialties, in primary as well as secondary care. The integration of the psychological and social dimensions of frailty could further enhance communication, allowing nonmedical professionals, such as social workers, to efficiently participate in the care for the older individual, in primary care and beyond.

\section{References}

1 Morley JE, Vellas B, Abellan van Kan G, Anker SD, Bauer JM, Bernabei R, et al. Frailty consensus: a call to action. JAMDA. 2013;14:392-7.

2 Rockwood K, Mitnitski A. Frailty defined by deficit accumulation and geriatric medicine defined by frailty. Clin Geriatr Med. 2011;27:17-26

3 Fried L, Tangen M, Walson J, Newman AB, Hirsch C, Gottdiener J, et al. Frailty in older adults: evidence for a phenotype. J Gerontol A Biol Sci Med Sci. 2001;56:M146-56.

4 Gobbens RJJ, Luijkx KG, Wijnen-Sponselee MT, Schols JMGA. Towards an integral conceptual model of frailty. J Nutr Health Aging. 2010;14:175-81.

5 Gobbens RJJ, van Assen MALM, Luijkx KG, WijnenSponselee MT, Schols JMGA. The Tilburg Frailty Indicator: psychometric properties. J Am Med Dir Assoc. 2010;11(5):34455 .

6 Collard RM, Oude Voshaar RC. Frailty; een kwetsbaar begrip. Tijdschr Psychiatr. 2013;54:59-69.

7 Panza F, Solfrizzi V, Frisardi V, Maggi S, Sancarlo D, Addante $\mathrm{F}$, et al. Different models of frailty in predementia and dementia syndromes. J Nutr Health Aging. 2011;15:711-9.

8 Andrew MK, Mitnitski AB, Rockwood K. Social vulnerability, frailty and mortality in elderly people. PLoS ONE. 2005;3: e2232.

9 http://epp.eurostat.ec.europa.eu

10 Wertelaers A, Govaerts F. Preventie van letsels tengevolge van vallen bij 65-plussers. Huisarts $\mathrm{Nu}$. 2001;30:434-52.

11 Collard RM, Boter H, Schoevers RA, Oude Voshaar RC. Prevalence of frailty in community-dwelling older persons: A Systematic review. J Am Geriatr Soc. 2012;60:1487-92.

12 Clegg A, Young J, Ilife S, Olde Rikkert M, Rockwood K. Frailty in elderly people. Lancet. 2013;381:752-62.

13 Morley JE, Malmstrom TK, Miller DK. A simple frailty questionnaire (FRAIL) predicts outcomes in middle aged African Americans. J Nutr Health Aging. 2012;16(7): 601-8.

14 Rockwood K. What would make a definition of frailty successful? Age Ageing. 2005;34:432-4.

15 Pialoux T, Goyard J, Lesourd B. Screening tools for frailty in primary health care: a systematic review. Geriatr Gerontol Int. 2012;12:189-97.

16 Xue QL, Beamer Ba, Chaves PHM, Guralnik JM, Fried LP. Heterogeneity in rate of decline in grip, hip and knee strength and the risk of all-cause mortality: the women's health and aging study II. J am Geriatr Soc. 2010;58:2076-84.

17 Abellan Van Kan G, Rolland Y, Houles M, Gillette-Guyonnet $\mathrm{S}$, Soto M, Vellas B. The assessment of frailty in older adults. Clin Geriatr Med. 2010;26:275-86.

18 Peters LL, Boter H, Buskens E, Slaets JPJ. Measurement properties of the Groningen Frailty Indicator in home-dwelling and institutionalized elderly people. J Am Med Dir Assoc. 2012;13:546-51.

19 Lacas A, Rockwood K. Frailty in primary care: a review of its conceptualization and implications for practice. BMC Med. 2012;10:4.

20 Drubbel I, Bleijenberg N, Kranenburg G, Eijkemans RJ, Schuurmans MJ, de Wit NJ, et al. Identifying Frailty: do the Frailty Index and Groningen Frailty Indicator cover different clinical perspectives? A cross-sectional study. BMC Fam Pract. 2013; $14: 64$ s.

21 Souriala N, Wolfsona C, Bergman H, Zhu B, Karunananthan $\mathrm{S}$, Quail $\mathrm{J}$, et al. A correspondence analysis revealed frailty deficits aggregate and are multidimensional. J Clin Epidemiol. 2010;63:647-54

22 Baitara A, Van Fraeyenhove F, Vandebroek A, De Droogh E, Galdermans D, Mebis J, et al. Evaluation of the Groningen Frailty Indicator and the G8 questionnaire as screening tools for frailty in older patients with cancer. J Geriatr Oncol. 2013;4:32-38.

23 Mitinski A, Fallah N, Rockwood MRH, Rockwood K. Transitions in cognitive status in relation to frailty in older adults: a comparison of three frailty measures. J Nutr Health Aging. 2011;15:863-7.

24 Subra J, Gillette-Guyonnet S, Cesari M, Oustric S, Vellas B. The integration of frailty into clinical practice: preliminary results from the Gérontopôle. J Nutr Health Aging. 2012;1:714-20.

25 Mcmillan GJ, Hubbard RE. Frailty in older inpatients: what physicians need to know. Q J Med. 2012;105:1059-65.

26 Robinson TN, Wallace JI, Wu DS. Accumulated frailty characteristics predict postoperative discharge institutionalization in the geriatric patient. J Am Coll Surg. 2011;213: $37-42$.

27 Ellis G, Whitehead MA, Robinson D, O'Neill D, Langhorne P. Comprehensive geriatric assessment for older adults admitted to hospital: meta-analysis of randomised controlled trials. BMJ. 2011;343:d6553.

28 Salvi F, Morichi V, Grilli A, Lancioni L, Spazzofumo L, Polonara S, et al. Screening for frailty in elderly emergency department patients by using the Identification of Seniors At Risk (ISAR). J Nutr Health Aging. 2012;6:314-9.

29 Bagshaw SM, McDermid RC. The role of frailty in outcomes from critical illness. Curr Opin Crit Care. 2013;19:496-503.

30 Extermann M, Aapro M, Bernabei R, Cohen HJ, Droz J, Lichtmanet $\mathrm{S}$, et al. Use of comprehensive geriatric assessment 
in older cancer patients: recommendations from the task force on CGA of the International Society of Geriatric Oncology (SIOG). Crit Rev Oncol Hematol. 2005;55:24152.

31 Armstrong JJ, Stolee P, Hirdes JP, Poss JW. Examining three frailty conceptualizations in their ability to predict negative outcomes for home-care clients. Age Ageing. 2010;39:755-8.
32 Siscovick DS, Fried L, Mittelmark M, Rutan G, Bild D, O'Leary DH. Exercise intensity and subclinical cardiovascular disease in the elderly. The Cardiovascular Health Study. Am J Epidemiol. 1997;145(11):977-86.

33 Taylor HL, Jacobs DR Jr, Schucker B, Knudsen J, Leon AS, Debacker G. A questionnaire for the assessment of leisure time physical activities. J Chronic Dis. 1978;31(12):741-55. 